\title{
Kuramoto model with time-varying parameters
}

\author{
Spase Petkoski and Aneta Stefanovska* \\ Department of Physics, Lancaster University, Lancaster LA1 4YB, United Kingdom
}

(Received 27 June 2011; published 23 October 2012)

\begin{abstract}
We analyze the Kuramoto model generalized by explicit consideration of deterministically time-varying parameters. The oscillators' natural frequencies and/or couplings are influenced by external forces with constant or distributed strengths. A dynamics of the collective rhythms is observed, consisting of the external system superimposed on the autonomous one, a characteristic feature of many thermodynamically open systems. This deterministic, stable, continuously time-dependent, collective behavior is fully described, and the external impact to the original system is defined in both the adiabatic and the nonadiabatic limits.
\end{abstract}

DOI: 10.1103/PhysRevE.86.046212

PACS number(s): 05.45.Xt, 87.10.Mn

\section{INTRODUCTION}

Biological examples provided the original motivation lying behind the Kuramoto model (KM) of coupled-phase oscillators [1]. However, neither the original model [2] nor any of its extensions [3] have incorporated a fundamental property of living systems - their inherent time variability. Many important characteristics of open systems can be missed by not accounting for the nonequilibrium dynamics that stems from their time-dependent (TD) parameters. Additionally, the application of the KM to many problems would move closer to reality by allowing for the natural frequency of each oscillator, or the coupling strengths, to be externally modulated by TD forcing, as commonly occurs in living systems. Among the numerous collective rhythms traceable back to TD parameters are the frequency flows in brain signals [4], modeling of brain dynamics under anesthesia [5] where the anesthetic strength modulates natural frequencies [6], event-related oscillatory responses of the brain [7], and dynamics of cardiovascular aging [8]. None of these are adequately described by existing models. Additionally, similar considerations are to be expected in nonbiological examples such as pattern formation in a nonlinear medium far from equilibrium [9]. Here, using trapped ions, one can vary the parameters at will and see the effect on synchronization.

There has already been much work on coupled oscillators influenced by noise as a special form of external dynamics [10]. Likewise, driving by an external periodic force [11] is a long-explored model, characterized by the interplay to the phases of each oscillator between the external pacemaker and the mean field of all other oscillators. A generalization of the KM that allowed certain time-varying frequencies and couplings was also numerically explored in [12]. However, the simulations were performed over a very small number of oscillators, the dynamics were not described analytically, and a qualitative description was not given for slow- or fast-varying cases. Other studies of nonconstant collective rhythms include asymmetrically coupled ensembles [13] and populations with multimodally distributed natural frequencies [14], with their complex mean field being a result of the multimodal distribution of the parameters. Frequency adaptation as discussed in [15] assumes nonconstant natural frequencies, but without an external influence. It is similar to the models with inertia [16], and its dynamics, apart from the stable incoherence, are characterized by either synchronization or a bistable regime of both synchronized and incoherent states. In addition, the model with drifting frequencies [17] assumes frequency dynamics formulated as an Ornstein-Uhlenbeck process, but it also leads to time-independent mean fields, resembling the simple KM under the influence of colored noise. Alternately switching connectivity [18] and periodic couplings [19] are some examples with varying coupling strengths. Yet, most of the discussions in these studies are concerned with the networks and graph theory properties of the system, and only Heaviside step functions are considered for the interaction between oscillators.

Nevertheless, the TD mean fields in most of these models result either from multistability or from unstable equilibria. Despite this, even in cases where it stems from some external system $[12,18,19]$, the low-dimensional mean-field dynamics and slow/fast reduced approaches are still missing. As such, none of these models can fully demonstrate the deterministic and stable TD dynamics of many real physical, chemical, biological, or social systems that can never be completely isolated from their surroundings. These systems do not reach equilibrium but, instead, exhibit complex dynamical behavior that includes the TD frequencies and couplings. We show that our generalization of the KM encompasses these dynamics.

\section{MODEL}

An external, explicitly TD, bounded function $x(t)$ is introduced. It modulates the frequencies or couplings of the original model. This external influence can also originate from another [20] nonconstant mean field. In the most general case, the strengths of the interactions $I_{i}$ are distributed according to a probability density function (PDF) $h(I)$ and, likewise, the distribution $g(\omega)$ of the natural frequencies $\omega_{i}$. Thus, depending on which parameter is influenced, two generalized KMs emerge.

$$
\begin{array}{ll}
\mathrm{A}: & \dot{\theta}_{i}=\omega_{i}+I_{i} x(t)+K r(t) \sin \left[\psi(t)-\theta_{i}\right], \\
\mathrm{B}: & \dot{\theta}_{i}=\omega_{i}+\left[K+I_{i} x(t)\right] r(t) \sin \left[\psi(t)-\theta_{i}\right] .
\end{array}
$$


Here, a TD complex order parameter is introduced,

$$
z(t)=r(t) e^{i \psi(t)}=\frac{1}{N} \sum_{j=1}^{N} e^{i \theta_{j}},
$$

where $r(t)$ and $\psi(t)$ are the TD mean-field amplitude and phase, respectively. For clarity, their explicit time dependence is henceforth omitted.

For each oscillator at any given time there is a 1:1 correspondence between the fixed and the TD parameters, i.e.,

$$
\tilde{\omega}_{i}(t)=\omega_{i}+I_{i} x(t)
$$

for model A and

$$
\tilde{K}_{i}(t)=K+I_{i} x(t)
$$

for model B, or in general,

$$
\tilde{I}_{i}(t)=I_{i} x(t) .
$$

Thus, for known forcing $x(t)$, a single oscillator from both nonautonomous (NA) models can be uniquely defined by the fixed parameters $\omega_{i}$ and $I_{i}$ or by the TD natural frequencies for model A and TD couplings for model B, $\tilde{\omega}_{i}$ and $\tilde{K}_{i}$, respectively, which in this case also encompass $x(t)$. Similarly, instead of $\tilde{\omega}_{i}$ and $\tilde{K}_{i}, \omega_{i}$ and $\tilde{I}_{i}$ can be used, whereas the distributions of these TD variables accordingly become $\tilde{g}(\tilde{\omega})$, $\tilde{\Gamma}(\tilde{K})$, and $\tilde{h}(\tilde{I})$.

To analyze models (1) and (2) the thermodynamic limit $N \rightarrow \infty$ is assumed. Here, the state of the system with fixed forcing $[x(t)=$ const] would have been described by a continuous PDF $\rho(\theta, \omega, I, t)$ which gives the proportion of oscillators with phase $\theta$ at time $t$, for fixed $\omega$ and $I$ [21]. On the other hand, the one-to-one correspondence between the fixed and the TD parameters in terms of PDFs implies that the same number of oscillators can be described by either of the following PDFs,

$$
|h(I) d I|=|\tilde{h}(\tilde{I}(I, t)) d \tilde{I}|
$$

or

$$
|g(\omega) d \omega|=|\tilde{g}(\tilde{\omega}(\omega, I, t)) d \tilde{\omega}|,
$$

and

$$
|\Gamma(K, I) d K|=|\tilde{\Gamma}(\tilde{K}(K, I, t)) d \tilde{K}|
$$

if $\tilde{\omega}$ and $\tilde{K}$ are used for describing the population. Also, the infinitesimal number of oscillators $d N$ is given by

$$
\begin{aligned}
d N & =|\rho(\theta, \omega, I, t) g(\omega) h(I) d \theta d \omega d I| \\
& =|\tilde{\rho}(\theta, \omega, \tilde{I}, t) g(\omega) \tilde{h}(\tilde{I}) d \theta d \omega d \tilde{I}|,
\end{aligned}
$$

where the PDFs $\rho$ and $\tilde{\rho}$ give the proportion of oscillators with phase $\theta$ at time $t$, for given fixed $\omega$ and $I$ or fixed $\omega$ and TD $\tilde{I}$, respectively. From probability theory it is known that, by definition, any PDF is non-negative, and by substituting (4) into (5) it directly follows that

$$
\rho(\theta, \omega, I, t)=\tilde{\rho}(\theta, \omega, \tilde{I}, t), \quad \text { where } \quad \tilde{I}=I x(t) .
$$

Analogously, for $\tilde{\omega}$ and $\tilde{K}$ instead of $\tilde{I}$, one would obtain

$$
\rho(\theta, \omega, I, t)=\tilde{\rho}_{1}(\theta, \tilde{\omega}, K, t)=\tilde{\rho}_{2}(\theta, \omega, \tilde{K}, t),
$$

with $\tilde{\omega}=\omega+I x(t)$ and $\tilde{K}=K+I x(t)$.
Thereafter, the state of the oscillatory system can be described either by a continuous PDF $\rho(\theta, \omega, I, t)$, which assumes fixed parameters, or by its counterpart $\tilde{\rho}(\theta, \omega, \tilde{I}, t)$, with TD parameters. However, since using the PDF with TD parameters would further complicate the continuity equation for a fixed volume by including gradients along the TD variables also, we choose to define the distribution for the fixed $\omega$ and $I$. In this way, the only gradient of the PDF $\rho$ is along the phases.

The chosen PDF $\rho$ is then normalized as

$$
\int_{0}^{2 \pi} \rho(\theta, \omega, I, t) d \theta=1 .
$$

Moreover, in the $\theta, \omega, I$ parameter space the number of oscillators given by $\rho(\theta, \omega, I, t) g(\omega) h(I) d \theta d \omega d I$ for each natural frequency $\omega$ and strength $I$ of the forcing $x(t)$ is conserved, and only phases $\theta$ change with time. Thus, the gradient along $\theta$ will be solely responsible for divergence of the oscillators. Hence the continuity equation for every fixed $\omega$ and $I$ is given by

$$
\begin{aligned}
& \text { A : } \frac{\partial \rho}{\partial t}=-\frac{\partial}{\partial \theta}\left\{\left[\omega+I x(t)+\frac{K}{2 i}\left(z e^{-i \theta}-z^{*} e^{i \theta}\right)\right] \rho\right\}, \\
& \text { В : } \frac{\partial \rho}{\partial t}=-\frac{\partial}{\partial \theta}\left\{\left[\omega+\frac{K+I x(t)}{2 i}\left(z e^{-i \theta}-z^{*} e^{i \theta}\right)\right] \rho\right\},
\end{aligned}
$$

where the velocity along $\theta$ is substituted from the governing equations (1) and (2). Definition (3) is also included in (7) and (8), rewritten using

$$
\frac{1}{N} \sum_{j} \sin \left(\theta_{j}-\theta_{i}\right)=\operatorname{Im}\left\{z e^{-i \theta_{i}}\right\},
$$

so that it becomes

$$
z=\int_{0}^{2 \pi} \int_{-\infty}^{\infty} \int_{-\infty}^{\infty} \rho(\omega, I, \theta, t) g(\omega) h(I) e^{i \theta} d \theta d \omega d I .
$$

The same reasoning for preserving the number of oscillators would also apply for $\tilde{\rho}(\theta, \omega, \tilde{I}, t) g(\omega) \tilde{h}(\tilde{I}) d \theta d \omega d \tilde{I}$ if the infinitesimal volume of the space $\theta, \omega, \tilde{I}$ is moving with $x(t)$ along the axis of the TD parameter, which, in this case, is $\tilde{I}$. Thus, again, the only gradient of $\tilde{\rho}$ would be along phases, and continuity equations would have the same form as (7) and (8), with $I x(t)$ substituted with $\tilde{I}$, and $\rho$ with $\tilde{\rho}$.

\section{LOW-DIMENSIONAL DYNAMICS}

Since $\rho(\theta, \omega, I, t)$ is real and $2 \pi$ periodic in $\theta$, it allows a Fourier expansion. The same would also hold for $\tilde{\rho}(\theta, \omega, \tilde{I}, t)$. Next, we apply the Ott and Antonsen ansatz [22] in its coefficients, such that

$$
f_{n}(\omega, I, t)=[\alpha(\omega, I, t)]^{n} .
$$

Thus,

$$
\rho(\theta, \omega, I, t)=\frac{1}{2 \pi}\left\{1+\left\{\sum_{n=1}^{\infty}[\alpha(\omega, I, t)]^{n} e^{i n \theta}+\text { c.c. }\right\}\right\},
$$


where c.c. is the complex conjugate. Substituting (10) into the continuity equations (7) and (8), it follows that this special form of $\rho$ is their particular solution as long as $\alpha(\omega, I, t)$ evolves with

$$
\begin{aligned}
& \mathrm{A}: \quad \frac{\partial \alpha}{\partial t}+i[\omega+\operatorname{Ix}(t)] \alpha+\frac{K}{2}\left(z \alpha^{2}-z^{*}\right)=0, \\
& \mathrm{~B}: \quad \frac{\partial \alpha}{\partial t}+i \omega \alpha+\frac{K+\operatorname{Ix}(t)}{2}\left(z \alpha^{2}-z^{*}\right)=0,
\end{aligned}
$$

for models A and B. The same ansatz as implemented in Eq. (9) reduces the order parameter to

$$
z^{*}=\int_{-\infty}^{+\infty} \int_{-\infty}^{+\infty} \alpha(\omega, I, t) g(\omega) h(I) d \omega d I .
$$

Equations (11) and (12) hold for any distributions of $\omega$ and $I$ and for any forcing $x(t)$. They describe the evolution of the parameter $\alpha$, which is related to the complex mean field through the integral equation, (13). These integrals can be analytically solved for certain distributions $g(\omega)$ and $h(I)$, thus directly leading to the low-dimensional evolution of $z$. Hereafter we focus on all such cases and therefore in all further analysis the natural frequencies follow a Lorentizan distribution, and $\alpha(\omega, I, t)$ is continued to the complex $\omega$ plane so $g(\omega)$ can be written as

$$
g(\omega)=\frac{1}{2 \pi i}\left[\frac{1}{\omega-(\hat{\omega}-i \gamma)}-\frac{1}{\omega-(\hat{\omega}+i \gamma)}\right],
$$

with poles $\omega_{p 1,2}=(\hat{\omega} \pm i \gamma)$, where $\hat{\omega}$ is the mean of $g(\omega)$.

\section{A. Time-dependent natural frequencies}

The simplest case of model A, Eq. (1), is when the external forcing is identical for each oscillator, $h(I)=\delta(I-\epsilon)$. This leads to trivial dynamics, solved by simply making the reference frame rotate at the TD frequency $\hat{\omega}+f(t)$, where $\hat{\omega}$ is the mean of $g(\omega)$ and $f+\dot{f} t=\epsilon x$.

The NA dynamics arises for nonidentical forcing. We first assume strengths proportional to frequencies, i.e.,

$$
\tilde{\omega}(t)=\omega[1+\epsilon x(t)]
$$

with a constant $\epsilon$. This means that $I=\epsilon \omega$ and $h(I)=g(\epsilon \omega)$, and since $\omega$ and $I$ in this case are not independent variables, the latter can be omitted in the PDF $\rho$. Hence, the integration in Eq. (13) is now only over $\omega$ and, by closing the integral in any of the complex half-planes, is given by the residue of the encircled pole. As a requirement from [22], $|\alpha(\omega, t)| \rightarrow 0$ as $\operatorname{Im}(\omega) \rightarrow \mp \infty$, depending on which pole is encircled. The last limit transforms Eq. (11) into $\frac{\partial \alpha}{\partial t}=-\tilde{\omega}(t) \alpha$. Thus, for $[1+\epsilon x(t)]>0$ the encircling is around the pole $\omega_{p 2}=(\hat{\omega}-$ $i \gamma)$, while for $[1+\epsilon x(t)]<0$ the upper-half-plane encircling involves $\omega_{p 1}=(\hat{\omega}+i \gamma)$. Next, the residue at these poles,

$$
z^{*}=\alpha(\hat{\omega} \mp i \gamma, t)
$$

is substituted in Eq. (11), yielding

$$
\dot{r}=-r\left[\gamma|1+\epsilon x(t)|+\frac{K}{2}\left(r^{2}-1\right)\right], \quad \dot{\psi}=\hat{\omega}[1+\epsilon x(t)] .
$$

Ansatz (10) holds only for nonidentical oscillators [26], implying the requirement $\tilde{\omega}(t) \neq 0, \forall t$.
If the previously discussed alternative continuity equation for $\tilde{\rho}$ was used, then $\alpha(\omega, I, t)$ would become $\tilde{\alpha}(\omega, \tilde{I}, t)$ and the poles of $\tilde{I}$ would be TD. Nevertheless, substituting $\tilde{\alpha}(\omega, I, t)$ into the continuity equation that includes $\tilde{I}$ would lead to the same evolution for the mean field, thus confirming the analysis.

Model A is also solvable with an independent Lorentzian distribution of forcing strengths. The frequencies follow $\tilde{\omega}(t)=\omega+I x(t)$ and the mean and half-width of $h(I)$ are $\hat{I}$ and $\gamma_{I}$, respectively. The integrals in Eq. (13) can again be closed in the lower or upper complex half-plane, and the requirements for $\alpha(\omega, I, t)$ are similar to those in the previous case. Hence, the $I$ integral for $x(t)>0$ is around the pole $I_{p 1}=\left(\hat{I}+i \gamma_{I}\right)$ and around $I_{p 2}=\left(\hat{I}-i \gamma_{I}\right)$ otherwise, while in the $\omega$ integral the encircling is around the pole $\omega_{p 2}=\hat{\omega}-i \gamma$. Thus, the residues give

$$
z^{*}=\alpha\left(\hat{\omega}-i \gamma, \hat{I}-i \gamma_{I}, t\right),
$$

which is applied in Eq. (11), so we finally obtain

$$
\dot{r}=-r\left[\gamma+\gamma_{I}|x(t)|+\frac{K}{2}\left(r^{2}-1\right)\right], \quad \dot{\psi}=\hat{\omega}+\hat{I} x(t) .
$$

A similar analysis would be possible for any other polynomial Lorentzian-like distributions of $\omega$ and $I$.

The only other analytically solvable form of model A that we are aware of is with multimodal $\delta$-distributed external strengths. For simplicity, we choose the bimodal function

$$
h(I)=\frac{1}{2}\left[\delta\left(I-\hat{I}-\gamma_{I}\right)+\delta\left(I-\hat{I}+\gamma_{I}\right)\right] .
$$

Integral (13) now leads to

$$
z^{*}=\frac{1}{2}\left[\alpha_{1}\left(\hat{\omega}-i \gamma, \hat{I}-\gamma_{I}, t\right)+\alpha_{2}\left(\hat{\omega}-i \gamma, \hat{I}+\gamma_{I}, t\right)\right],
$$

with dynamics consistently described by the evolutions of $\alpha_{1,2}$ obtained from Eq. (11),

$$
\begin{aligned}
\frac{\partial \alpha_{1,2}}{\partial t}= & -\left\{i\left[\hat{\omega}+\left(\hat{I} \mp \gamma_{I}\right) x(t)\right]-\gamma\right\} \alpha_{1,2} \\
& +\frac{K}{4}\left[\alpha_{1}+\alpha_{2}-\alpha_{1,2}^{2}\left(\alpha_{1}+\alpha_{2}\right)^{*}\right] .
\end{aligned}
$$

This case of model A was also investigated in [23], where Choi et al. carried out a bifurcation analysis near the limit $r K \ll 1$.

Following the restrictions on $x(t)$ in the problems analyzed in Fig. 1, we took

$$
x(t)=\cos \Omega t \quad \text { and } \quad \epsilon<1
$$

in the case of a strength proportional to the frequency, while for model A with independent Lorentzianly distributed strengths, the forcing is

$$
x(t)=1+\cos \Omega t .
$$

Finally, for bimodal $\delta$-distributed strengths, the absence of restrictions on the external field allows it to be the $x$ component of a Rössler oscillator [24]. In all the problems shown, the NA TD dynamics is revealed and fully described by the reduced NA low-dimensional system. A Runge-Kutta 4 algorithm was used for numerical integration of Eqs. (1) and (2) over 100000 oscillators, with a time step of $0.0025 \mathrm{~s}$, while the half-width and mean of the natural frequencies were $\gamma=1$ and $\hat{\omega}=0$, except where stated otherwise. 

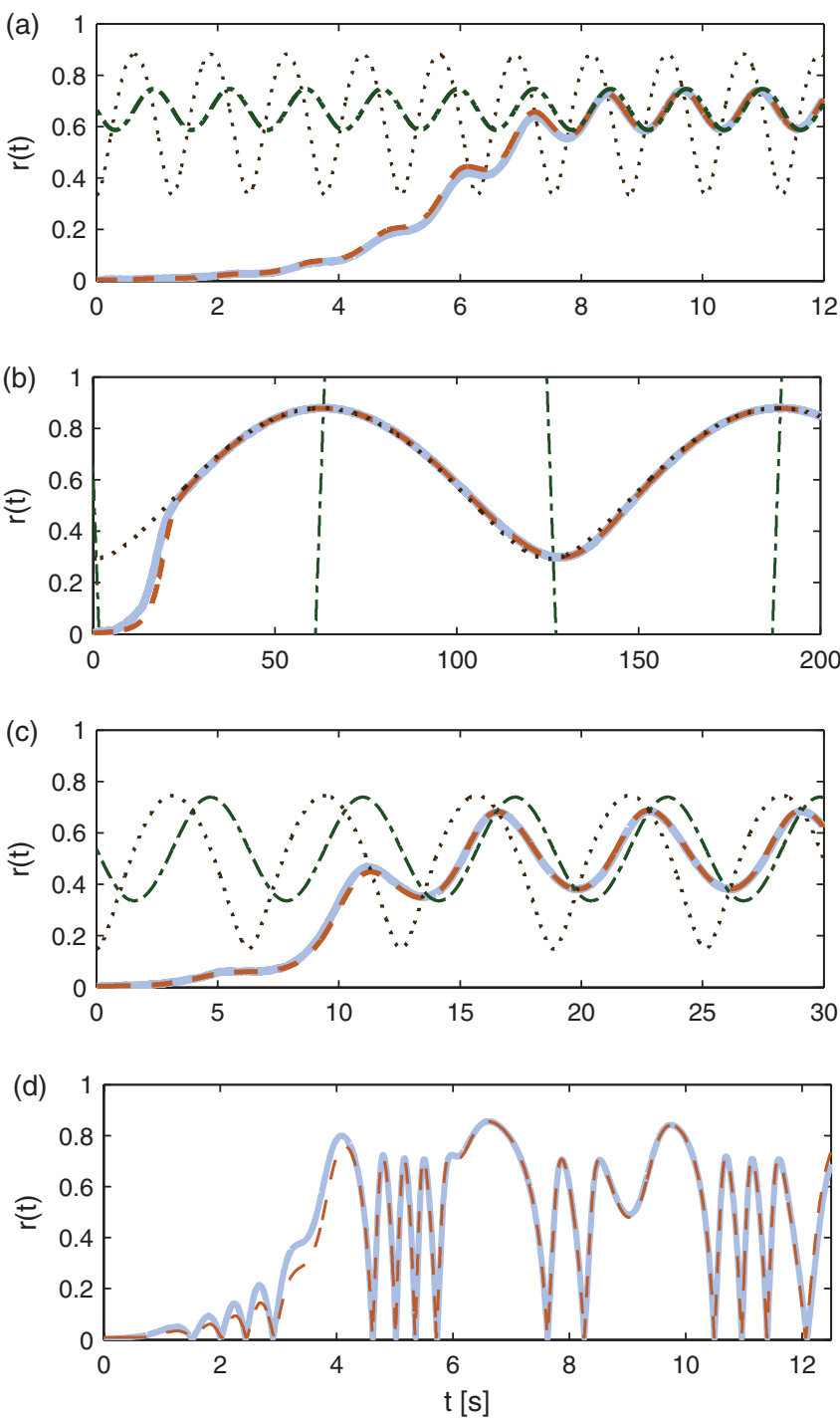

FIG. 1. (Color online) The time-varying mean field for model A, Eq. (1), resembles the externally applied cosine (a)-(c) or chaotic forcing (d). Numerical simulations of the full system, Eq. (1) [thick (light blue) curve], are in agreement with the low-dimensional dynamics [dashed (red) curve], Eqs. (14)-(17) (see text for details). Adiabatic [dotted (brown) curve] and nonadiabatic evolutions [dashed-dotted (green) curve], Eqs. (23)-(27), confirm the reduced dynamics in its limits (see text for details). The distribution $h(I)$ is (a), (b) the same as $g(\omega), K=3.5, \epsilon=0.6, \Omega=5$, and $\Omega=0.05$, respectively; (c) independent Lorentzian, $K=4.5, \gamma_{I}=0.6$ and $\Omega=1$; and (d) bimodal $\delta, K=8, \gamma=1, \gamma_{I}=1$, and $\hat{I}=1$.

\section{B. Time-dependent coupling strengths}

We have also investigated the low-dimensional evolution of NA model B, Eq. (2). Since all the couplings in the original model are equal, there is no qualitative difference between the situation with identical forcing to each coupling and that with coupling-dependent forcing. We chose the latter and proceed as for model A, yielding

$$
\dot{r}=-r\left[\gamma+\frac{K}{2}[1+\epsilon x(t)]\left(r^{2}-1\right)\right], \quad \dot{\psi}=\hat{\omega} .
$$

The analysis for multimodal $\delta$-distributed strengths is also very similar to that for model A, (1). For example, for bimodal $h(I)$, Eq. (16) holds again with $\alpha_{1,2}$ evolving as

$$
\begin{aligned}
\frac{\partial \alpha_{1,2}}{\partial t}= & -(i \hat{\omega}-\gamma) \alpha_{1,2}+\frac{1}{4} K\left[1+\left(\hat{I} \mp \gamma_{I}\right) x(t)\right] \\
& \times\left[\alpha_{1}+\alpha_{2}-\alpha_{1,2}^{2}\left(\alpha_{1}+\alpha_{2}\right)^{*}\right] .
\end{aligned}
$$

However, for a Lorentzian distribution $h(I)$, contour integration cannot be applied to Eq. (13). Namely, the integration contour should be such that if $\alpha(\omega, I, t)$ is analytic and $|\alpha| \leqslant 1$ everywhere inside the contour at $t=0$; this would also hold for all $t>0$. However, for this to happen, one of the requirements from [25] is $|\alpha| \leqslant 0$, for $|\alpha|=1$. This should be taken with regard to the semicircular integration path $I=|I| e^{i \vartheta}$ with $|q| \rightarrow \infty$ and $\vartheta \in(0, \pi)$ or $\vartheta \in(-\pi, 0)$ depending on the half-plane of the contour. Thus, substituting for $I$ into Eq. (12) and taking $|\alpha|=1$, it yields

$$
\frac{\partial|\alpha|}{\partial t}=|I| x(t) r \sin \vartheta \sin [\phi(\omega, I, t)-\psi(t)] .
$$

Here, $\phi$ is the phase of $\alpha$ that depends on $\omega, I$, and $t$, implying that the last sine can have either sign. Consequently, it cannot be proven that the condition $\frac{\partial|\alpha|}{\partial t} \leqslant 0$ holds $\forall t$ and $\omega$ on either of the half-planes. As a result, the integral in Eq. (9) cannot be solved for $I$ using the residue theorem.

In contrast, the restrictions do not affect the NA parts of the other discussed variations of model B. To confirm this generality, $x(t)$ for the problem shown in Fig. 2(b) is a chaotic signal from a Rössler oscillator. Similarly, the chosen amplitude of the cosine forcing in Fig. 2(a) allows close-to-incoherent dynamics to be observed in some intervals, so that the limitations of the slow-fast approaches discussed in the following section appear.

A theorem in [26] states that Eqs. (11) and (12) asymptotically capture all macroscopic behavior of the system as $t \rightarrow \infty$. Moreover the incoherent and partly synchronized states both belong to the manifold defined by Eqs. (11) and (12) [22], and the initial incoherent state is set with uniformly distributed phases at time $t=0$. Thereafter, the ansatz, (11) and (12), and the evolutions, (14)-(19), should continuously describe our system, as confirmed by Figs. 1 and 2 .

\section{REDUCED DYNAMICS}

The plots in Figs. 1(a)-1(c) and 2(a) show that the oscillations of the mean field follow the frequency of the external forcing, but this raises the questions of what the amplitude of the oscillations is and whether they can adiabatically follow the forcing. Similarly, an obvious feature of the same results is the low-frequency filtering of the external fields, i.e., the only difference between Fig. 1(a) and Fig. 1(b) is the frequency of the external forcing, while its influence is much more prominent in the latter. This is actually a well-known, but not much explored, characteristic of population models, and it is a direct consequence of their intrinsic transient dynamics [1].

In the following we adopt fast-slow reduction to simplify the evolution for simple periodic forcing. The reduction depends on the period of the external field $T=2 \pi / \Omega$, relative to the system's transition time, $\tau$, and has not been applied to similar systems. The exponential damping rate of the original system 

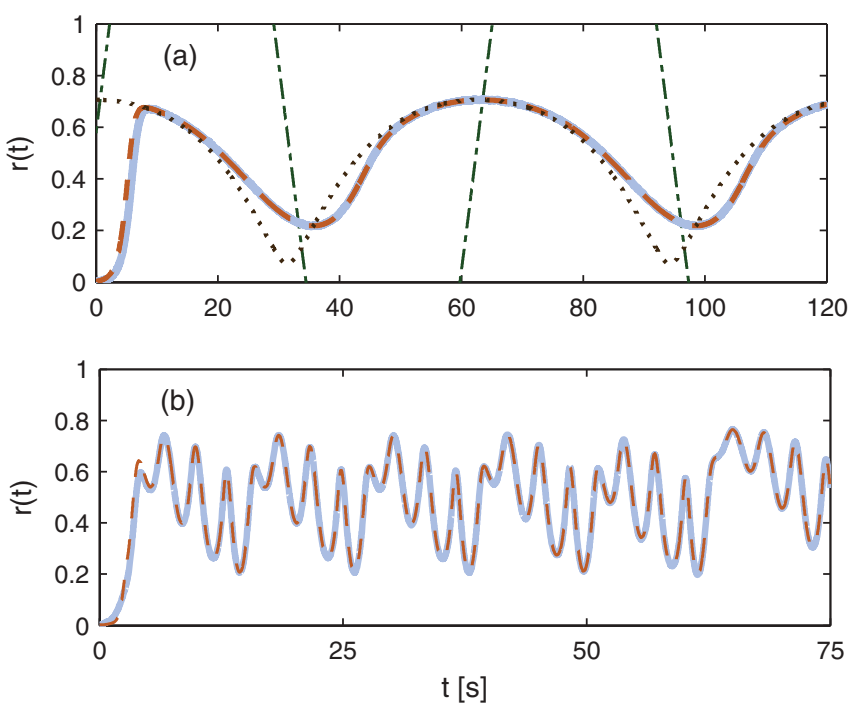

FIG. 2. (Color online) Time-varying mean field for model B, Eq. (2), follows the external cosine (a) or the chaotic (b) forcing. The numerical simulation of Eq. (2) [thick (light blue) curve] coincides with the low-dimensional evolution [dashed (red) curve], Eq. (18), shown in (a), and Eq. (19), shown in (b). (a) Adiabatic [dotted (brown) curve], Eq. (26), and nonadiabatic evolution [dashed-dotted (green) curve], Eq. (25), for constant forcing with $K=3, \Omega=0.1$, and $\epsilon=0.33$. (b) Bimodal $\delta$-distributed strengths with $K=5, \gamma_{I}=1$, and $\hat{I}=0$.

is defined by $\tau$ [22] and

$$
\tau=1 /|K / 2-\gamma| .
$$

For a system far from incoherence, $K=2 \gamma+O(2 \gamma), \tau \approx$ $1 / O(\gamma)$ holds, meaning that the transition time depends only on the width of the distribution of natural frequencies. Thereafter for this case, the system's response is adiabatic for slow external fields, $\Omega \ll \gamma$, and nonadiabatic for fast fields, $\Omega \gg \gamma$. Henceforth, the dependence on $\gamma$ is removed by scaling the time and the couplings in the autonomous system, $t=t / \gamma, K=K / 2 \gamma$, and $\tau=1 /|K-1|$ (the scaled variables keep the same letters).

For model A, Eq. (1), with $x(t)=\cos \Omega t$ and $h(I)=\delta(I-$ $\epsilon)$, after the initial transition and in the absence of bifurcations, the amplitude of the mean field consists of a constant term $r_{0}$ and a TD term $\Delta r(t)$. For the nonadiabatic response, simulations [thick (light blue) lines in Fig. 1(a)] show that $\Delta r(t) \sim 1 / \Omega$ and $r_{0} \gg \Delta r(t)$. Thereafter $r_{0}$ can be expressed as averaged over one period $T=2 \pi / \Omega$ of the oscillations of $\Delta r(t)$. This way it follows that $0=-r_{0}+K\left(r_{0}^{3}-r_{0}\right)$ [27], or

$$
r_{0}=\sqrt{1-1 / K}
$$

Further, we apply $r(t) \approx r_{0}$ and $\frac{d r}{d t}=\frac{d \Delta r}{d t}$ to Eq. (14) and then integrate it. From there

$$
\Delta r(t)=-r_{0} \frac{\epsilon}{\Omega} \sin \Omega t,
$$

and the magnitude of the NA response is

$$
\Delta_{\text {fast }}=2 \frac{\epsilon}{\Omega} \sqrt{1-\frac{1}{K}} .
$$

Hence the long-term nonadiabatic evolution follows

$$
r_{\text {fast }}(t)=\sqrt{1-\frac{1}{K}}\left(1-\frac{\epsilon}{\Omega} \sin \Omega t\right) .
$$

The adiabatic behavior emerges through the introduction of a slow time scale $t^{\prime}=\Omega t$, such that the system is constant on the fast time scale $t$ and changes only in $t^{\prime}$. Hence the left-hand side of Eq. (14) is 0, whence

$$
r_{\text {slow }}(t)=\sqrt{1-\frac{1+\epsilon \cos \Omega t}{K}},
$$

while, for the magnitude of the NA part, we obtain

$$
\Delta_{\text {slow }}=\sqrt{1-\frac{1-\epsilon}{K}}-\sqrt{1-\frac{1+\epsilon}{K}} .
$$

An analogous analysis can be performed for the appropriate form of model B, Eq. (2), leading to the low-dimensional evolution for fast cosine forcing given by

$$
r_{\text {fast }}(t)=\left(1+\frac{\epsilon}{\Omega} \sin \Omega t\right) \sqrt{1-\frac{1}{K}},
$$

and for slow forcing,

$$
r_{\text {slow }}(t)=\sqrt{1-\frac{1}{K(1+\epsilon \cos \Omega t)}} .
$$

For the dynamics of model A with independent Lorentzian strengths and cosine forcing, $x(t)=1+\cos \Omega t$, the time is scaled by $\left(\gamma+\gamma_{I}\right)$, and the dynamics follows

$$
r_{\text {fast }}(t)=\sqrt{1-\frac{1}{K}}\left[1-\frac{\gamma_{I}}{\Omega\left(\gamma+\gamma_{I}\right)} \sin \Omega t\right]
$$

for fast forcing, while for slow driving,

$$
r_{\text {slow }}(t)=\sqrt{1-\frac{1}{K}-\frac{\gamma_{I} \cos \Omega t}{K\left(\gamma+\gamma_{I}\right)}} .
$$

The adiabatic responses can also be obtained from the selfconsistency of Eqs. (7) and (9) for stationary states of the mean field. Namely, assuming very slow dynamics of the external forcing, the system can be treated as quasistationary. This is similar to assuming stationarity on a fast time scale. Thus one obtains

$$
r=\sqrt{1-2 \gamma(t) / K(t)}
$$

which corresponds to results (23) and (26).

All the evolutions for reduced dynamics [Figs. 1(a)-1(c) and 2(a)] are in line with the above analysis, confirming the interplay between external and internal time scales of the NA system. The magnitudes of the slow/fast responses to cosine forcing are given in Fig. 3 for model A, Eq. (1), with forcing strengths following the frequencies' distribution. They confirm the obtained dependence of $\Delta$ on the frequency and amplitude of the external field. The low-frequency filtering mentioned before is also obvious. The transient behavior for slow and fast forcing can be seen in Fig. 3(b), where $\Delta$ is shown for both the actual and the reduced dynamics. This plot perfectly 

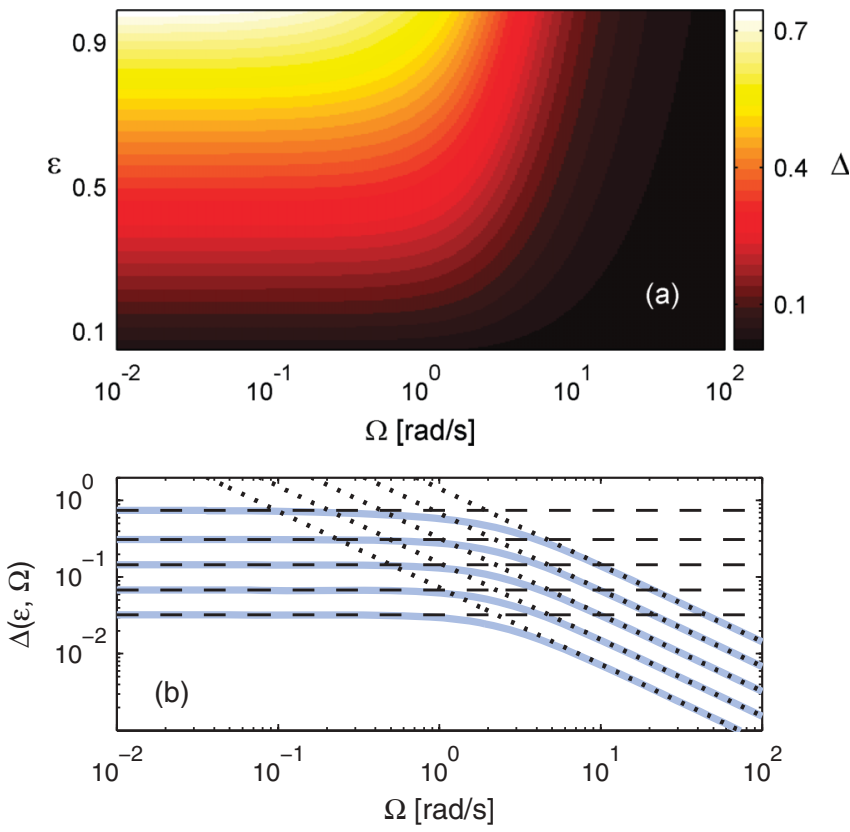

FIG. 3. (Color online) Magnitude of the response, $\Delta(\epsilon, \Omega)$, of the NA model A to cosine forcing, Eq. (1). External forcing strengths follow the distribution of frequencies, $K=4.25$ and $\Omega \in\left[10^{-2}, 10^{2}\right]$. (a) Results from Eq. (14) for $\epsilon \in[0.05,0.99]$. (b) Nonadiabatic [dotted (black) lines], Eq. (21), and adiabatic [dashed (black) lines], Eq. (24), evolution for $\epsilon \in\{0.05,0.1055,0.2225,0.4693,0.99\}$, compared with the real dynamics [thick (light blue) lines], Eq. (14).

matches the analytic limits for application of the reduction approaches. Similar plots can also be obtained for the other problems analyzed. However, for coupling close to critical, the system's transition time increases and for $K \approx K_{c}, \tau \rightarrow \infty$. As a result, the slow dynamics fails, as shown in Fig. 2(a) at the minima of $r$ when it is close to 0 , unlike the case $K=$ $K_{c}+O\left(K_{c}\right)$ given in Figs. 1(a) and 1(b) or Fig. 2(a) for $r$ far from 0 .

\section{DISCUSSION}

With the analysis of the reduced dynamics, supplementing the full low-dimensional description, all aspects of the TD KM have been demonstrated. The former is shown only for simple periodic forcing, but this does not decrease the generality of the reduction, since any external field can be represented by its Fourier components. These methods are of great importance in modeling systems with multiple time scales of oscillation and interaction, such as the human cardiovascular system [28] and inhibitory neurons in the cortex [29].

In summary, we have characterized a new dynamics of interacting oscillators subject to continuous, deterministic perturbation. It consists of the dynamics of an external system superimposed on the original collective rhythm and was missing from earlier models [3], possibly leading to an incorrect interpretation of some real dynamical systems. We have derived the impact of the forcing and evaluated the effect of its dynamics, amplitude, and distribution. Thus, we have proposed a generalization of the KM that encompasses NA systems [30] and is directly applicable to any thermodynamically open system. For example, the observed time variations of brain dynamics can be easily explained as a consequence of TD frequencies or couplings of the single neurons, where the source of the external variation could be due to anaesthesia [5], event related [7], or due to some influence from another part of the brain. In particular, the stable, time-varying mean field can now be reconstructed, and in this way, a large range of systems tackled by the $\mathrm{KM}$ - spanning from a single cell up to the level of brain dynamics — can be described more realistically.

\section{ACKNOWLEDGMENTS}

We thank P. V. E. McClintock and G. Lancaster for useful comments on the manuscript, and A. Duggento, L. Basnarkov, Y. Suprunenko, and D. Iatsenko for valuable discussions. The work was supported by the Engineering and Physical Sciences Research Council (UK) [Grant No. EP/100999X1] and by a Lancaster University PhD grant.
[1] S. Strogatz, Sync: The Emerging Science of Spontaneous Order (Hyperion, New York, 2003).

[2] Y. Kuramoto, Chemical Oscillations, Waves, and Turbulence (Springer-Verlag, Berlin, 1984).

[3] J. A. Acebrón, L. L. Bonilla, C. J. Pérez Vicente, F. Ritort, and R. Spigler, Rev. Mod. Phys. 77, 137 (2005); S. H. Strogatz, Physica D 143, 1 (2000); A. Pikovsky, M. Rosenblum, and J. Kurths, Synchronization - A Universal Concept in Nonlinear Sciences (Cambridge University Press, Cambridge, 2001).

[4] D. Rudrauf et al., Neuroimage 31, 209 (2006).

[5] J. H. Sheeba, A. Stefanovska, and P. V. E. McClintock, Biophys. J. 95, 2722 (2008).

[6] B. Musizza, A. Stefanovska, P. V. E. McClintock, M. Paluš, J. Petrovčič, S. Ribarič, and F. F. Bajrović, J. Physiol. 580, 315 (2007).

[7] G. Pfurtschelle and F. H. Lopes da Silva, Clin. Neurophysiol. 110, 1842 (1999).
[8] Y. Shiogai, A. Stefanovska, and P. V. E. McClintock, Phys. Rep. 488, 51 (2010).

[9] T. E. Lee and M. C. Cross, Phys. Rev. Lett. 106, 143001 (2011).

[10] S. H. Strogatz and R. E. Mirollo, J. Stat. Phys. 63, 613 (1991).

[11] S. Shinomoto and Y. Kuramoto, Prog. Theor. Phys. 75, 1105 (1986); H. Sakaguchi, ibid. 79, 39 (1988).

[12] D. Cumin and C. Unsworth, Physica D 226, 181 (2007).

[13] E. Montbrió, J. Kurths, and B. Blasius, Phys. Rev. E 70, 056125 (2004); J. H. Sheeba, V. K. Chandrasekar, A. Stefanovska, and P. V. E. McClintock, ibid. 79, 046210 (2009).

[14] L. L. Bonilla, J. C. Neu, and R. Spigler, J. Stat. Phys. 67, 313 (1992); J. A. Acebrón, L. L. Bonilla, S. De Leo, and R. Spigler, Phys. Rev. E 57, 5287 (1998).

[15] D. Taylor, E. Ott, and J. G. Restrepo, Phys. Rev. E 81, 046214 (2010).

[16] J. A. Acebrón and R. Spigler, Phys. Rev. Lett. 81, 2229 (1998).

[17] J. Rougemont and F. Naef, Phys. Rev. E 73, 011104 (2006). 
[18] P. So, A. Bernard, B. C. Cotton, and E. Barreto, Chaos 18, 037114 (2008); S. P. Kuznetsov, A. Pikovsky, and M. Rosenblum, ibid. 20, 043134 (2010).

[19] S. H. Lee, S. Lee, S.-W. Son, and P. Holme, Phys. Rev. E 85, 027202 (2012).

[20] J. H. Sheeba, V. K. Chandrasekar, and M. Lakshmanan, Phys. Rev. E 84, 036210 (2011).

[21] R. Mirollo and S. H. Strogatz, J. Nonlinear Sci. 17, 309 (2007).

[22] E. Ott and T. M. Antonsen, Chaos 18, 037113 (2008).

[23] M. Y. Choi, Y. W. Kim, and D. C. Hong, Phys. Rev. E 49, 3825 (1994).

[24] O. E. Rössler, in Synergetics: a Workshop: Proceedings of the International Workshop on Synergetics at Schloss Elmau, Bavaria (May 2-7, 1977), edited by H. Haken (Springer, Berlin, 1977), p. 174; Phys. Lett. A 57, 397 (1976).
[25] E. Montbrió and D. Pazó, Phys. Rev. Lett. 106, 254101 (2011).

[26] E. Ott and T. M. Antonsen, Chaos 19, 023117 (2009).

[27] This is obtained from averaging both sides of Eq. (14) for one period. The term $\Delta r(t) \cos \Omega t$ in the integral vanishes only if $\Delta r(t) \sim \sin \Omega t$, which is self-consistently proved, as the obtained form of $\Delta r(t)$ for nonadiabatic response, Eq. (22), follows this assumption.

[28] A. Stefanovska and M. Bračič, Contemp. Phys. 40, 31 (1999).

[29] B. Ermentrout and M. Wechselberger, SIAM J. Appl. Dyn. Syst. 8, 253 (2009).

[30] M. Rasmussen, Attractivity and Bifurcation for Nonautonomous Dynamical Systems, Lecture Notes in Mathematics No. 1907 (Springer, New York, 2007). 